\title{
Künstliche Intelligenz und Zeitmaschine
}

\section{Jean Martin}

Dr. med., Mitglied der Redaktion

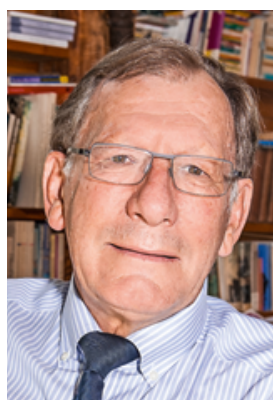

\section{Literatur \\ 1 Docteur 4.0 (Begeg nung mit Dr. Antoine Geissbuhler). Le Temps, 13. Dezember 2016, S. 22. Garelli Stéphane. Qui doit craindre l'intelli- gence artificielle? Le Temps (Lausanne), 10. Dezember 2016, S. 16 Kaplan Frédéric. L’Eu- rope doit construire la première Time Machine. Le Temps, 15. Dezember 2016, S. 10.}

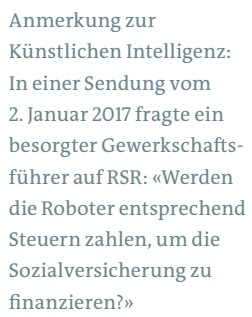

jean.martin[at]saez.ch
Der in Cambridge lehrende Physiker Stephen Hawking sagte der BBC: «Die Entwicklung der Künstlichen Intelligenz könnte das Ende der Menschheit bewirken. Der Mensch ist begrenzt durch eine langsame biologische Evolution und könnte nicht mit den sich schnell immer wieder neu programmierenden Maschinen Schritt halten ...» Ich spiele zwar nicht in derselben Liga wie Hawking, doch diese in unterschiedlicher Geschwindigkeit verlaufende Anpassung beschäftigt mich stark. Sie beschäftigt auch A. Geissbuhler, Leiter der Abteilung für E-Health der HUG in Genf [1]. Ist unser Organismus mental (und auch biologisch) in der Lage, sich so schnell anzupassen? Man könnte argumentieren, dass Tablets bedienende Dreijährige in dieser Hinsicht beruhigen sollten. Vielleicht.

Aus Überzeugung oder ideologischen Gründen wollen wir nicht glauben, dass Maschinen intelligenter sein werden, als der Mensch. S. Garelli, Professor an der Universität Lausanne, erinnert jedoch daran, dass Maschinen dank der immensen Datenmenge seit dem Jahr 1995 die Informationsvielfalt mit ihren Algorithmen viel besser auswerten können [2]. 1997 wurde Garry Kasparov vom IBM-Rechner 'Deeper Blue' im Schach geschlagen und vor kurzem gewann ein anderer Rechner gegen einen grossen Meister im Go-Spiel. Künstliche Intelligenz kann sich ständig neu programmieren und dabei auf Big Data zurückgreifen.

10-Tausend(Milliarden?)-Dollar-Frage: Wird die Künstliche Intelligenz eines Tages in der Lage sein, wie Baudelaire zu dichten, wie Descartes oder Kant zu philosophieren - oder diese gar zu übertreffen? Ich habe den Eindruck, dass immer mehr Menschen geneigt sind, diesen Satz nicht mehr als Frage aufzufassen. Geissbuhler ist «hin- und hergerissen zwischen der Gewissheit, dass die Maschine eine Vormachtsstellung hat, und der Schöpferkraft des Menschen [...] Zurück zum Menschen mit seiner Empathie, seiner Intuition und seinen Zweifeln, vor denen jeder Roboter verstummt?» F. Kaplan, Professor für den Bereich Digital Humanities am Eidgenössischen Polytechnikum Lausanne (EPFL), will Europa die erste Time Machine bringen [3]. Das Vorhaben beeindruckt. Es gilt eine einzigartige Speicherund Rechner-Infrastruktur aufzubauen, mit deren
Hilfe die Daten der Vergangenheit abgebildet, an die Gegebenheiten der Gegenwart angepasst und auf die Zukunft projiziert werden». Internet et al. entwickelten sich, ohne die zeitliche Komponente wirklich einzubeziehen, als 'grosses Hier und Jetzt'. Aber die auf die Sekunde genau dokumentierte Gegenwart ist bereits das Archiv ihrer eigenen Zukunft ... An dieser Stelle gilt es anzumerken, dass Facebook und Google die Verwalter unseres unwiderruflich privatisierten Menschheitserbes sein werden. Wollen wir das?

Die zweite Internetrevolution beginnt mit dem Auftritt der Zeit als Indexierungsprinzip. Es geht in der Folge nicht mehr um eine punktuelle Archivierung, sondern um einen Brückenschlag zwischen dem globalisierten Informationszeitalter und den vorausgegangenen Epochen. «Diese Big Data der Vergangenheit dokumentieren dann unser kulturelles, aber auch unser wirtschaftliches, wissenschaftliches und technisches Welterbe. [...] So können neue Interpretationsmodelle geschaffen werden. Die Epidemiologen werden die Verbreitung verheerender Krankheiten besser verstehen, die Ökonomen werden Wachstum und Krisen des Kapitalismus neu interpretieren, die Linguisten die Verflechtungen unserer Sprachen.» So wird - sagt Kaplan - «die Vergangenheit zu bewohnbarem Territorium. Künstliche Intelligenz ermöglicht das Erschliessen vergangener, nicht dokumentierter Räume, verfolgt die Handlungswege der damaligen Akteure und verleiht ihnen eine emotionale Dimension.»

Wie wohl jeder von uns träumte ich gelegentlich davon, einmal einen Tag im alten Rom, im Mittelalter, bei den Inkas oder den Sioux vor der Conquista erleben zu können. Dort sind wir jetzt - oder bald - mit der Zeitmaschine. Kaplan: «Wir werden morgen Zeitreisen unternehmen können. Das Projekt 'Time Machine' bietet einzigartige Möglichkeiten, unsere Zukunft auf der Grundlage eines gemeinsamen Erbes zu gestalten.» Diese Perspektive hätte Churchill zu schätzen gewusst. Er meinte dazu «Der menschliche Geist ist nur wenig dafür gerüstet, der unendlichen Komplexität der Phänomene gerecht werden zu können» und «Je mehr wir in die Vergangenheit blicken können, desto weiter sehen wir in die Zukunft.»" 